\title{
Natural variation in life history and aging phenotypes is associated with mitochondrial DNA deletion frequency in Caenorhabditis briggsae
}

\author{
Suzanne Estes ${ }^{1 *}$, Anna L Coleman-Hulbert ${ }^{1 \dagger}$, Kiley A Hicks ${ }^{1 \dagger}$, Gene de Haan ${ }^{1}$, Sarah R Martha', Jeremiah B Knapp ${ }^{1}$,
} Samson W Smith ${ }^{1}$, Kevin C Stein ${ }^{1}$, Dee R Denver ${ }^{2}$

\begin{abstract}
Background: Mutations that impair mitochondrial functioning are associated with a variety of metabolic and age-related disorders. A barrier to rigorous tests of the role of mitochondrial dysfunction in aging processes has been the lack of model systems with relevant, naturally occurring mitochondrial genetic variation. Toward the goal of developing such a model system, we studied natural variation in life history, metabolic, and aging phenotypes as it relates to levels of a naturally-occurring heteroplasmic mitochondrial ND5 deletion recently discovered to segregate among wild populations of the soil nematode, Caenorhabditis briggsae. The normal product of ND5 is a central component of the mitochondrial electron transport chain and integral to cellular energy metabolism.

Results: We quantified significant variation among C. briggsae isolates for all phenotypes measured, only some of which was statistically associated with isolate-specific ND5 deletion frequency. We found that fecundity-related traits and pharyngeal pumping rate were strongly inversely related to ND5 deletion level and that C. briggsae isolates with high ND5 deletion levels experienced a tradeoff between early fecundity and lifespan. Conversely, oxidative stress resistance was only weakly associated with ND5 deletion level while ATP content was unrelated to deletion level. Finally, mean levels of reactive oxygen species measured in vivo showed a significant non-linear relationship with ND5 deletion level, a pattern that may be driven by among-isolate variation in antioxidant or other compensatory mechanisms.
\end{abstract}

Conclusions: Our findings suggest that the ND5 deletion may adversely affect fitness and mitochondrial functioning while promoting aging in natural populations, and help to further establish this species as a useful model for explicit tests of hypotheses in aging biology and mitochondrial genetics.

\section{Background}

Caenorhabditis elegans has long been appreciated as a useful model organism for developmental, molecular, and aging biology. As molecular genetic and genomic approaches have become increasingly available, nematode biologists have also become interested in explaining and predicting natural patterns of phenotypic and genomic evolution. These interests have motivated recent efforts to quantify natural phenotypic variation and molecular population genetic structuring, and to characterize the ecology and natural history of nematode

\footnotetext{
* Correspondence: estess@pdx.edu

† Contributed equally

'Department of Biology, Portland State University, Portland, OR 97201, USA

Full list of author information is available at the end of the article
}

species in order to interpret such findings within robust population and ecological genetic contexts [e.g., [1-5]]. Caenorhabditis briggsae was until recently the closest known relative of $C$. elegans and has therefore been the subject of many different types of comparative analyses [e.g., [6-9]]. Building upon previous discoveries of mitochondrial DNA (mtDNA) variation segregating among natural populations $[10,11]$, we sought to further develop the C. briggsae system by quantifying the degree to which this mtDNA diversity is associated with population-level variation in life-history, physiological, and aging related phenotypes. This work will provide a foundation for generating and testing hypotheses in mitochondrial mutation biology and aging research.
C Biomed Central

(c) 2011 Estes et al; licensee BioMed Central Ltd. This is an Open Access article distributed under the terms of the Creative Commons Attribution License (http://creativecommons.org/licenses/by/2.0), which permits unrestricted use, distribution, and reproduction in any medium, provided the original work is properly cited. 
Mutations that impair functioning of the mitochondrial electron transport chain (ETC) are associated with a variety of human metabolic and age-related disorders [12-14]. A barrier to in-depth studies of the inheritance, population dynamics, and effects of clinically relevant mtDNA mutations has been the lack of genetic model systems that adequately represent the features of pathogenic mtDNA mutations [14]. Howe and Denver [10] discovered a naturally occurring heteroplasmic mtDNA deletion segregating among geographically distinct populations of the soil nematode, Caenorhabditis briggsae. The deletion eliminates the 5' end (first $786 \mathrm{bp}$ ) of the NADH dehydrogenase subunit 5 (ND5) gene, the normal protein product of which is an essential and highly conserved core subunit of mitochondrial ETC complex I [10]. In humans, several neurodegenerative disorders including Parkinson's disease are associated with heteroplasmic ND5 mutations [13].

The $C$. briggsae deletion occurs as a consequence of directly repeated $21 \mathrm{bp}$ DNA sequence tracts in the ND5 gene and in an upstream pseudogene named $\Psi N D 5-2$ (Figure 1A). The observed deletion is expected to strongly and negatively affect ND5 protein-coding function as the deleted sequences encode more than 200 ND5 amino acids, 34 of which are conserved in C. elegans, D. melanogaster, and humans. Two PCR approaches showed that deletion-containing genomes constitute from 0 to $\sim 50 \%$ of total within-isolate mitochondrial populations (Figure 1B, Additional file 1, Table S1).

Based on other studies of ETC complex I-deficient genotypes [15-18], we hypothesized that expression of truncated ND5 protein products would be associated with mitochondrial dysfunction, increased reactive oxygen species (ROS) production, reduced fitness, and faster rates of aging. Higher rates of nuclear [6,19] and mitochondrial [20] mutation in C. briggsae relative to C. elegans and a negative correlation between ND5 deletion frequency and reproductive output in C. briggsae [10] are consistent with this hypothesis, although it cannot yet be ruled out that nuclear-encoded genes are responsible instead.

With the dual goals of further characterizing global phenotypic variation in C. briggsae and developing a novel model system for mitochondrial mutation dynamics, ETC dysfunction, and aging research, we performed a survey of natural variation in fitness, physiological, and aging related phenotypes among $C$. briggsae isolates containing different proportions of ND5-deletion bearing mtDNA genomes. We found significant among-isolate variation in all phenotypes studied, some of which was statistically associated with isolate-specific ND5 deletion heteroplasmy level. Specifically, C. briggsae isolates containing a high proportion of ND5 deletion genomes tended to have reduced
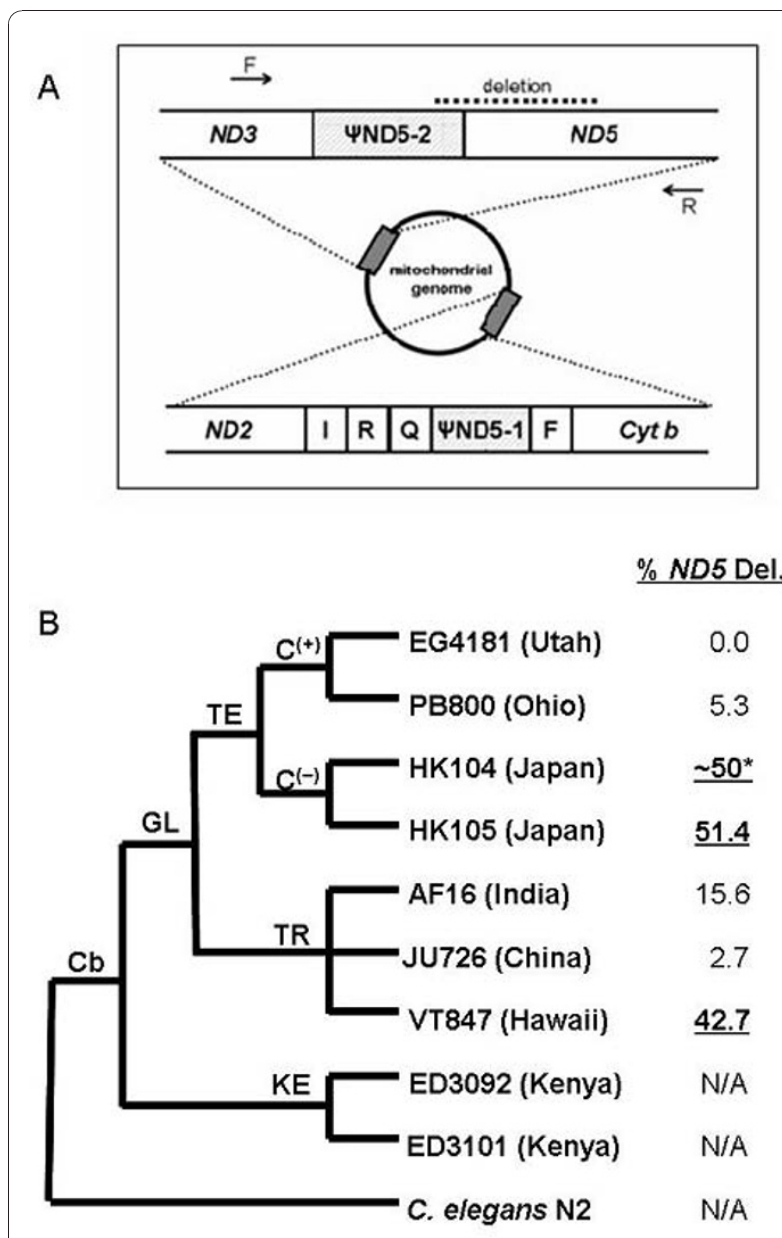

Figure 1 C. briggsae mitochondrial ND5 deletion. A. C. briggsae mitochondrial genome positions of ND5 deletion (dashed line at top) and $\Psi$ ND5 elements-pseudogenes that originated from duplications of ND5. Arrows = primers for PCR assays [reproduced from [10]]. B. C. briggsae intraspecific phylogeny with C. elegans as outgroup. $\mathrm{GL}=$ global intraspecific superclade; $\mathrm{KE}=$ Kenya clade; $\mathrm{TE}$ and $\mathrm{TR}=$ temperate and tropical subclades of $\mathrm{GL} . \mathrm{C}(+)=$ temperate-clade isolates bearing DRSeq2 compensatory YND5-2 alleles; C(-) = those bearing ancestral alleles. \% ND5 Del. indicates isolate-specific percentages of total mitochondrial genomes that harbor ND5 deletions as previously determined by qPCR [10] and confirmed by conventional PCR and gel analysis here (Additional file 1, Table S1). Isolates classified for statistical analyses as experiencing high (bold underlined font), low (normal font), or zero-ND5 ("N/A") deletion levels are indicated on the phylogeny. *ND5 deletion levels for the HK104 isolate used here was estimated based on the standard PCR band size assay described in Howe and Denver [10] where the results of the standard PCR assay were shown to correlate positively and significantly with a qPCR method used to estimate deletion levels in the other isolates.

reproductive fitness and early-onset physical decline compared to isolates with low or zero deletion levels. However, the same isolates did not differ consistently with respect to lifespan, ATP content, or acute oxidative stress resistance. Additionally, although the C. briggsae isolate with the 
highest ND5 deletion frequency exhibited the highest levels of ROS as expected, we found a non-linear relationship between ND5 deletion and ROS level. These patterns may be explained by as yet unknown differences in physiological adaptations among C. briggsae isolates; e.g., ROS detoxification mechanisms and reliance on anaerobic energy metabolism.

\section{Results and Discussion}

We surveyed variation in life history, physiology, and aging phenotypes among wild $C$. briggsae isolates that represent the full spectrum of natural variation in ND5 deletion frequency (Figure 1B). We found strong and significant negative correlations between ND5 deletion level and both nematode fecundity and population growth rate $(r)$ (Table 1$)$. These patterns are driven entirely by two of the three high-deletion isolates as compared to low and zero-deletion isolates (Figure 2A) and may indicate that the ND5 deletion has a negative effect on C. briggsae fitness only when its frequency reaches a certain threshold [cf. [21]]. Specifically, when isolates were subdivided into deletion frequency categories (Figure 1B legend), we found that high-ND5 deletion isolates had significantly reduced total fecundities and $r$ compared to either low or zero-deletion isolates (Tukey HSD, $\alpha=0.05$; Figure 2A). We also found a significant negative correlation between ND5 deletion level and lifespan (Table 1, Figure 2B), but the pattern is far weaker than that for fecundity. Indeed, low-deletion isolates were found to live longer than either high or zero-deletion isolates (Tukey HSD, $\alpha=0.05$ ), a pattern driven by the particularly long lifespan of low-deletion isolate, PB800 (Ohio) (Figure 2B).

Consistent with findings from C. elegans [e.g., [22,23]], we found little evidence for life history tradeoffs in C. briggsae isolates; average correlations between all pairs of reproduction and longevity traits were positive (Additional file 2, Table S2). However, we found one exception to this trend for isolate-specific trait correlations: the three
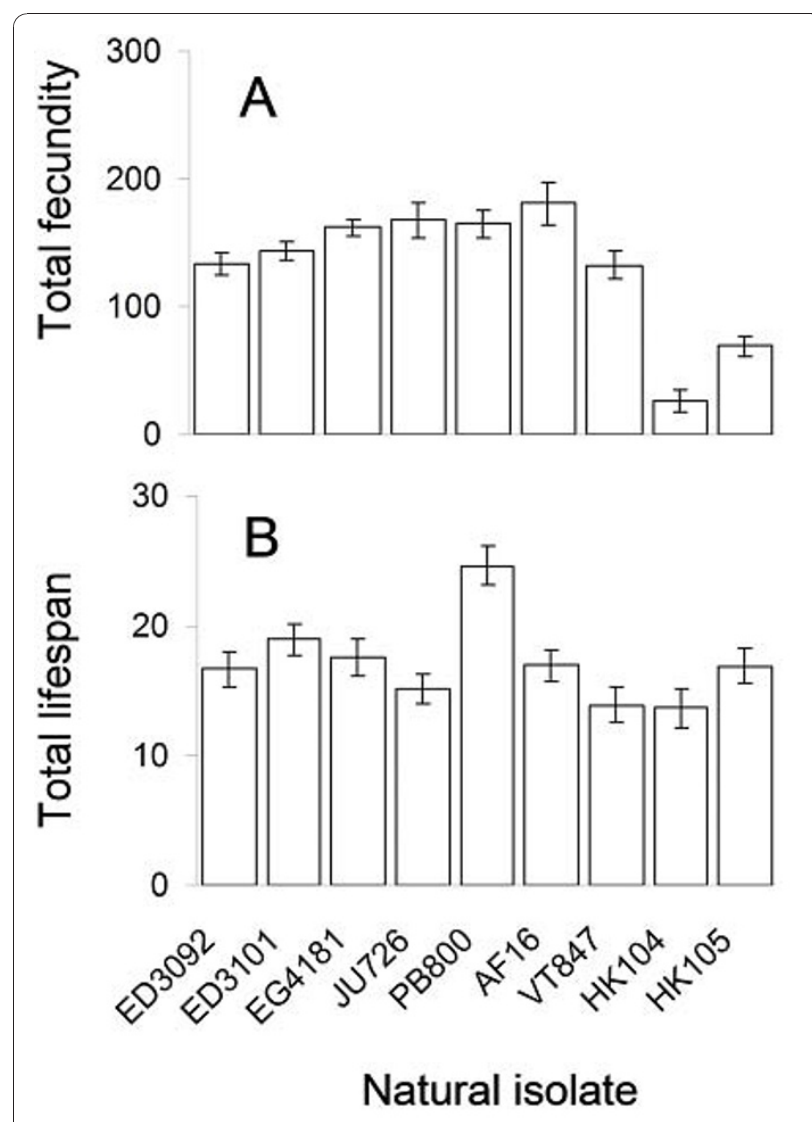

Figure 2 Natural variation in life history traits. Natural variation in mean lifetime fecundity (A) and mean lifespan (B) among $C$. briggsae isolates. Isolates are arranged along the $x$-axis in order of increasing ND5 deletion level. Bars show one standard error.

high-ND5 deletion C. briggsae isolates are significantly more likely to experience a tradeoff between early fecundity and longevity; i.e., animals that live long tend to have reduced fecundity early in life (Figure 3 ) when such a reduction will most negatively affect population growth rates. These findings are reminiscent of those for

Table 1 Phenotypic variation among C. briggsae isolates

\begin{tabular}{|c|c|c|c|c|c|c|}
\hline Character & Grand mean & SD & $\mathbf{N}$ & df & $\mathbf{F}$ & $\rho$ \\
\hline Early fecundity & 31.99 surviving offspring & 20.86 & 210 & 8 & $13.26^{* * *}$ & $-0.432^{* *}$ \\
\hline Late fecundity & 103.8 surviving offspring & 58.03 & 210 & 8 & $14.93^{* * *}$ & $-0.482^{* *}$ \\
\hline Total fecundity & 135.7 surviving offspring & 66.74 & 210 & 8 & $22.89^{* * *}$ & $-0.554^{* * *}$ \\
\hline Intrinsic rate of increase $(r)$ & 0.808 & 0.250 & 210 & 8 & $17.93^{* * *}$ & $-0.426^{* * *}$ \\
\hline Total lifespan & 16.96 days & 6.358 & 160 & 8 & $5.192^{* * *}$ & $-0.228^{* * *}$ \\
\hline Pharyngeal pumping & $181.6 \mathrm{pumps} / \mathrm{min}$ & 79.99 & 110 & 8 & $8.215^{* * *}$ & $-0.582^{* * *}$ \\
\hline ATP content & $4.499 \mathrm{nM} / \mathrm{mg}$ protein & 1.545 & 85 & 8 & $25.09^{* * *}$ & -0.120 \\
\hline Paraquat resistance & $61.03 \mathrm{~min}$ & 21.70 & 127 & 8 & $7.139^{* * *}$ & $-0.204^{* * *}$ \\
\hline Superoxide level & 224.20 relative fluorescence units & 18.55 & 99 & 8 & $8.388^{* * *}$ & 0.119 \\
\hline
\end{tabular}

Grand means, standard deviations, and sample sizes for all traits measured. $F$ is the test statistic for one-way analyses of variance for each phenotype in C. briggsae isolates. $\rho$ is the Pearson correlation coefficient between isolate-specific trait means and deletion frequencies. ${ }^{*},{ }^{* *}$, and ${ }^{* * *}$ denote significance at the $0.05,0.01$, and 0.001 levels, respectively. 


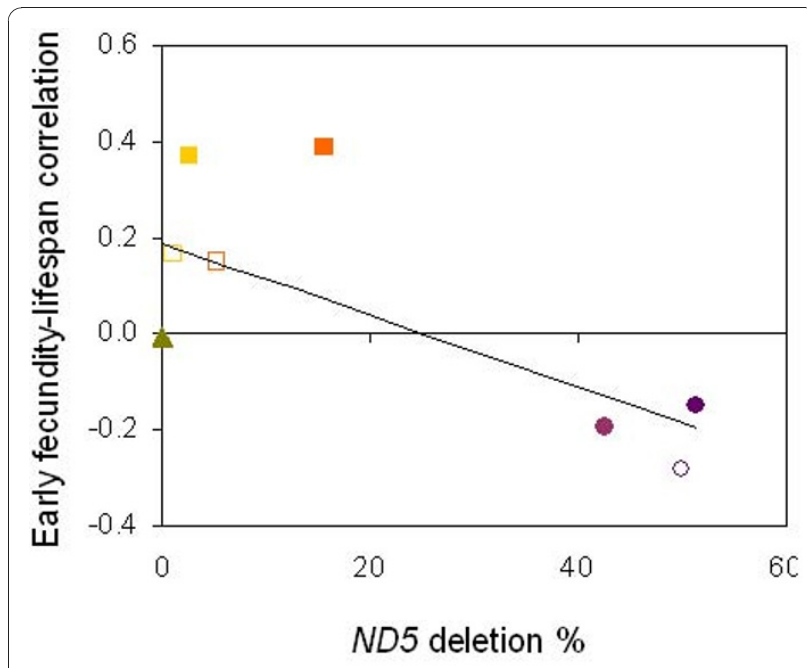

Figure 3 Relationship between life-history tradeoffs and ND5 deletion frequency. Linear regression of Spearman's correlation coefficients $(\rho)$ between early fecundity and total lifespan for each C. briggsae isolate on isolate-specific ND5 deletion level. Slope (SE) = -0.0074 (0.0029), $p=0.036$. Isolates are grouped as follows: zero deletion isolates ED3092 = open green triangle and ED3101 = filled green triangle (overlaps ED3092); low deletion isolates EG4181 = gold open square, JU726 = gold filled square, PB800 = orange open square, AF16 = orange filled square; and high deletion isolates VT847 = lavender filled circle, HK104 = purple open circle and HK105 = purple filled circle.

C. elegans long lived mutants assayed either under nutritional stress [22] or benign conditions [24] and are consistent with the idea that the tradeoffs predicted by life history theory [25] may only manifest themselves in nematodes experiencing some form of stress - either exogenous or endogenous stress (e.g., mitochondrial dysfunction).

Because pharyngeal pumping rate is a reliable biomarker of age in C. elegans [26,27], we hypothesized that highND5 deletion C. briggsae isolates would exhibit slower pharyngeal pumping in non-aging individuals as well as faster declines in pharyngeal pumping (i.e., increased muscle deterioration) with age when compared to low and zero-ND5 deletion isolates. In agreement with the first expectation, we observed a striking negative relationship between ND5 deletion level and average pharyngeal pumping rates in non-aging worms (Table 1, Figure 4). This trend persisted with increasing age; however, high deletion strains exhibited a somewhat slower rate of decline in pharyngeal pumping rates compared to low and zero-deletion isolates (Tukey HSD, $\alpha=0.05$, based on the difference in average pumping rate between days 2 and 20 post-L4; Figure 4). This may mean that high deletion isolates experience late-life reductions in their rates of aging compared to other isolates. Finally, we found significant natural variation in adult pharyngeal pumping rates throughout early but

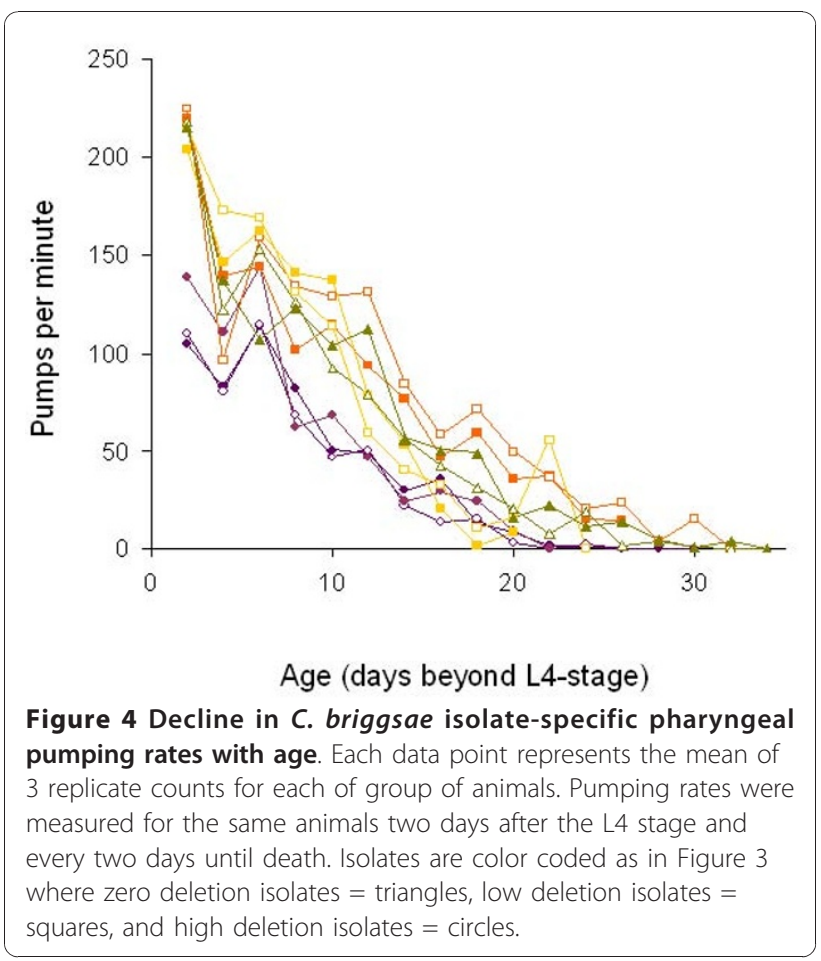

not late life (F-tests, $\mathrm{p}<0.05$ for time points prior to 14 days post-L4; analyses stopped after L $4+20$ days due to the reduction in statistical power caused by mortality). The magnitude of among-isolate differences in pumping rates was greatest during the young adult stage (Table 1; Figure 4) and declined slightly over the lifespan of the worms. Interestingly, the sharp decline in mean pumping rates at 4 days post-L 4 coincided with the end of the reproductive period; this pattern is not observed in the congener, C. elegans [e.g., [26], pers. obs.].

To further quantify natural phenotypic variation and to test whether the observed relationships between fitness-related life history traits and ND5 deletion levels may be mediated by mitochondrial ETC functioning, we quantified the relationship between ND5 deletion level and several aspects of animal physiology. Our expectation was that, as a result of reduced mitochondrial ETC efficiency, higher-ND5 deletion isolates would exhibit reduced levels of ATP and oxidative stress resistance, together with increased ROS production compared to lower or zero-ND5 deletion isolates.

Only some of the above expectations were borne out by the data. We found significant among-isolate variation in average ATP content of young adult animals; however, there was only a weak, nonsignificant negative relationship between ATP content and ND5 deletion frequency (Table 1, Figure 5A). If high-ND5 deletion isolate, VT847 (Hawaii), which produced $6.379 \pm 0.164$ $\mathrm{nM} \mathrm{ATP} / \mathrm{mg}$ protein, was removed from the analysis, 


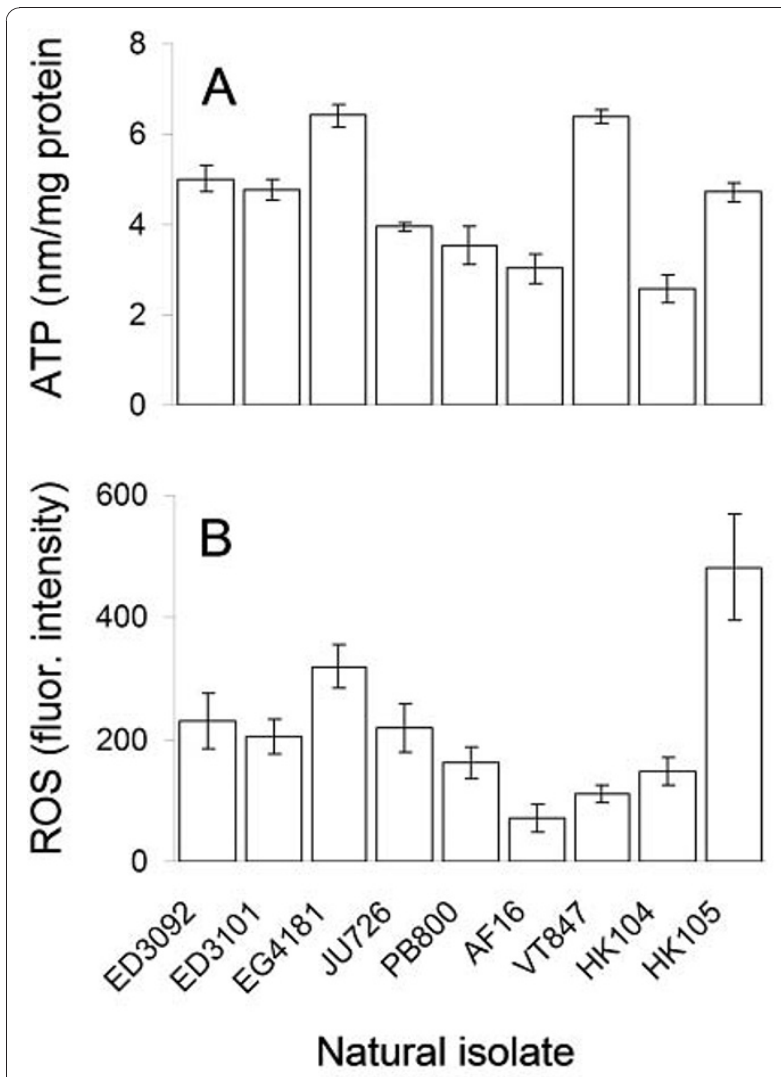

Figure 5 Natural variation in ATP and ROS levels. Natural variation in mean ATP content (A) and mean relative ROS levels in pharyngeal bulbs (B) among C. briggsae isolates. Isolates are arranged along the $x$-axis in order of increasing ND5 deletion level. Bars show one standard error. A second order quadratic provided the best fit to the relationship between relative superoxide level and ND5 deletion frequency (B), [adjusted $R^{* * *}=0.191$, coefficient $(S E)=0.451$ (0.093), $p<0.0001$ ] even if the HK105 isolate was omitted from the analysis [adjusted $R^{* *}=0.250$, coefficient $(S E)=$ $0.256(0.064), p<0.0001]$.

the correlation became more strongly negative (Pearson $\mathrm{r}=-0.370, \mathrm{p}=0.001)$. ATP production is known from other studies to sometimes be robust to ETC dysfunction; however, these data highlight the need to understand the degree to which nematodes may rely on alternate energy production pathways [28-30], which could obfuscate any pattern between ATP level and ND5 deletion frequency, for example.

We found significant among-isolate variation for oxidative stress resistance as measured by survival times of worms during acute paraquat exposure (Table 1). We predicted that, if higher-ND5 deletion isolates experience mitochondrial dysfunction leading to increased endogenous ROS levels, these isolates would be less able to survive attack from exogenous sources of ROS. A weak but significant negative relationship existed between this form of oxidative stress resistance and
ND5 deletion frequency (Table 1). This pattern was largely driven by two low-ND5 deletion isolates (PB800 and JU726) with high oxidative stress resistance (data not shown). We currently have no information on whether C. briggsae isolates vary in their expression of antioxidant proteins and thus in their abilities to detoxify ROS, which would provide one possible explanation for the weak relationship between paraquat-induced oxidative stress resistance and ND5 deletion heteroplasmy level.

Finally, relative ROS levels measured for pharyngeal bulbs also varied significantly among $C$. briggsae isolates (Table 1; Figure 5B). We measured total levels of mitochondrial oxidants using MitoSOX Red (Invitrogen). MitoSOX Red is preferentially taken up by actively respiring mitochondria, and while it is nearly exclusively oxidized by superoxide [31,32]-the most common form of mitochondrially generated ROS-it is considered an accurate and effective system for measuring total ROS levels in vivo [32]. In contrast to our simple expectation that a positive, linear relationship would exist between ND5 deletion frequency and ROS level, we found a significant, non-linear relationship between these variables such that zero and high deletion isolates tended to exhibit higher superoxide levels than low deletion isolates (Figure 5B legend). The in vivo fluorogenic dye-based method used here gives a view of net ROS level in living tissue and therefore reflects both the rate at which ROS are generated by cells and the rate at which they are scavenged by superoxide dismutases and other antioxidant systems. Consequently, we do not yet know which of these sources of variability explain more of the variance in net ROS levels among isolates, which was considerable despite modest sample sizes (Table 1). This variation suggests that $C$. briggsae may provide a useful system in which to test whether endogenous ROS have a role in generating variability in spontaneous mutation rates [33].

Thus, while more work is required to understand whether and how the ND5 deletion directly affects mitochondrial functioning in C. briggsae, it is clear that there is no simple relationship between isolate-specific net ROS production and the detrimental phenotypes associated with high ND5 deletion heteroplasmy levels in nature. For example, both Japanese isolates, HK104 and HK105, exhibit low fitness (Figure 2) and pharyngeal pumping rates (Figure 4), but their relative levels of superoxide (and ATP) are quite different from one another (Figure 5). These results may indicate that C. briggsae isolates experience different forms of mitochondrial dysfunction [Figure 3 in [34]] that may or may not be related directly to the NDS deletion.

The limited molecular population genetic structuring of C. briggsae [[35], but see [5]] belies the striking 
phenotypic variation among isolates reported here, the latter of which suggests an immense capacity for evolution of aging and life-history phenotypes. Together with previous findings of geographic variation in mitochondrial genome content among C. briggsae isolates [10] and of elevated spontaneous mutation rates in some isolates relative to the C. elegans N2 lab strain $[6,19,20]$, the findings from this paper suggest that $C$. briggsae will provide an opportunity to understand the linkages between mitochondrial function (e.g., ROS production), mutation processes, and aging. And importantly, because C. briggsae contains naturally-occurring mtDNA variation that is likely to affect organismal fitness, this system offers a major advantage for studying the evolutionary dynamics of mtDNA mutations over C. elegans where the majority of mtDNA mutant strains have been isolated from laboratory mutagenesis screens.

\section{Conclusions}

Although we found no straightforward relationship between ND5 deletion frequency and relative ROS levels, our accumulated findings are consistent with the notion that the natural mitochondrial genetic variation observed among C. briggsae isolates is detrimental to the organism. This is particularly true in light of recent studies that show high mutation rates in high-ND5 C. briggsae isolates $[6,19,20]$, Nevertheless, two issues prevent us from demonstrating that the ND5 deletion is the root cause of the detrimental phenotypes: 1) ND5 deletion level is clearly confounded with phylogenetic relationship (e.g., two of our three "high-deletion" isolates are sister taxa from Japan; Figure 1), and 2) we cannot disentangle the effects of among-isolate variation in ND5 deletion level from among-isolate nuclear variation on the phenotypes studied here. However, the ability to generate inbred lines and mitochondrial-nuclear hybrid strains in which mitochondria from natural C. briggsae isolates with different ND5 deletion heteroplasmy levels may be evaluated on uniform nuclear genetic backgrounds [36] will be a major advantage of this nematode system. (A large-scale analysis of such hybrid strains will be reported elsewhere.) A congener of the well-known model nematode, C. elegans, C. briggsae has a similar life-history and mating system, and offers many of the same advantages as C. elegans as an animal genetic model system [37]. Together with the ample among-isolate variation in ND5 deletion heteroplasmy level (Figure 1B) [10] and aging-related phenotypes, we contend that the C. briggsae system offers a powerful and versatile model for understanding the interplay between mitochondrial dysfunction and aging in an evolutionary context, as well as the basic biology underlying mtDNA deletion genetics. This study lays the necessary groundwork to rigorously test these and other questions in aging biology and life history theory [e.g., [38]].

\section{Methods}

Nematode strains and culture conditions

As described in [10], the $\Psi N D 5-2$ pseudogene directly upstream of ND5 (Figure 1A) is required for the ND5 deletions to occur within $C$. briggsae. Directly repeated nucleotide sequences occur within ND5 and $\Psi N D 5-2$ that promote direct repeat-associated deletion events. Isolates in the "Kenya" clade (Figure 1B) provide natural outgroup controls because they lack $\Psi N D 5-2$ and are thus inherently unable to experience the ND5 deletions. The among-isolate variability in average heteroplasmy level is thought to also be partly accounted for by the presence of compensatory sequences within the mtDNA of certain populations (Figure 1B legend). These sequences, present at the $\Psi N D 5-2$ direct repeat associated with the deletions, appear to prevent the deletion by reducing sequence homology between $\Psi N D 5-2$ and $N D 5$ and to thereby place an upper limit on the proportion of ND5-deletion bearing genomes able to accumulate within individuals [10]. We chose to use a subset of nine natural C. briggsae isolates studied by [10] that captured the full range of ND5 deletion heteroplasmy level (Figure 1, Additional file 1, Table S1). All nematodes were grown under standard laboratory conditions on 15 mm plates with NGM and Escherichia coli strain HB101. All nine natural isolates were included in each analysis.

\section{Life-history assays}

Isolate-specific fecundities were estimated for each natural isolate following [39]. "Early fecundity" is the number of offspring produced on the first two days of reproduction combined; "late fecundity" is the number of offspring produced on the third and fourth days of reproduction. We also calculated total lifespan as the number of days lived from the egg stage. We calculated intrinsic rate of increase, $r$, for each natural isolate by solving $\sum e^{* * * *} l(x) m(x)$ for $r$, where $l(x)$ is the proportion of worms surviving to day $x$ and $m(x)$ is the fecundity on day $x$. We also quantified average correlations among life history traits across C. briggsae isolates to investigate whether the magnitude or pattern of these trait associations would change with increasing ND5 deletion frequency.

\section{Pharyngeal pumping}

We measured the decline of pharyngeal pumping rate with age among $C$. briggsae isolates. Caenorhabditid nematodes ingest soil bacteria using rhythmic contractions of the pharynx - a neuromuscular organ comprising 20 muscle cells and 20 neurons. As in humans, 
muscle organization and function in nematodes deteriorates with chronological age. The rate of pharyngeal pumping is known to decline steadily with age in C. elegans as a result of contraction-related cellular injury $[27,40]$. Pharyngeal pumping was quantified for the same 10 to 20 individuals per isolate beginning two days after the L4 stage, and every other day until death. Daily pharyngeal pumping rates were taken as the average of three 5 -second counts, converted to pumps per minute.

\section{ATP content}

ATP content was measured using a protocol adapted from [[41], B. Braeckman, pers. comm.] for 8-10 independent, age-synchronous samples of young adult nematodes from each isolate. ATP content was determined for $50 \mathrm{uL}$ of supernatant using the ATP Bioluminescence Assay Kit CLS II (Roche) and following the manufacturer's instructions. ATP measurements were standardized by total protein content using a BCA Kit (Pierce).

\section{Oxidative stress resistance}

We measured resistance of $C$. briggsae to acute paraquat exposure following [42]. Age-synchronized populations of post-reproductive ( 8 day old) worms were generated for each natural isolate. Sixteen individuals per isolate were exposed to $300 \mathrm{mM}$ paraquat in S-basal and scored for survival every 5 minutes. Worms that remained completely unresponsive after three taps with a glass prod were scored as dead.

\section{Relative superoxide production}

We followed the basic approach of [31] to quantify relative ROS levels among C. briggsae isolates. Briefly, agesynchronized worms were incubated for 24 hours in 10 uM MitoSOX Red (Invitrogen) before being transferred as young adults to fresh NGM plates seeded with nonlabeled HB101 E. coli. They were allowed to feed for 1 hour, and paralyzed using a drop of $5 \mathrm{M}$ levamisole prior to imaging. Images were acquired using a high resolution wide field Core DV system (Applied Precision ${ }^{\mathrm{TM}}$ ), equipped with an Olympus IX71 inverted microscope mounted with a Nikon Coolsnap ES2 HQ camera (Advanced Light Microscopy Core Facility, Oregon Health and Science University, Portland, OR). Fluorescent, z-stack images of the mitochondrial-rich pharynx with a 1.0 second exposure time were captured at $60 \mathrm{X}$ magnification. Images were deconvolved prior to analysis. Terminal pharyngeal bulbs were manually circled to quantify mean fluorescence intensity of the area in exposed and unexposed (control) animals for each strain using ImageJ software (NIH). The final pharyngeal bulb intensity values were calculated as the difference between intensity values for exposed and control worms.
Although the use of mitochondria-specific dyes has become a widely used and accepted method for measuring a variety of mitochondrial traits in vivo, a caveat is that differential dye uptake between samples could lead to inaccurate interpretation of fluorescence differences. There is currently no means of determining the extent to which this occurs [31]. However, great care was taken to minimize all other sources of error. For example, we exposed only one sample at a time to the microscope light source and used the shortest exposure time possible to avoid introducing variation due to breakdown of the dye.

\section{Statistical analyses}

We analyzed among-isolate variation using separate one-way analyses of variance (ANOVA) for each phenotype measured. To test for differences between pairs of isolates, least-squares contrasts (Tukey's HSD for all pairwise comparisons; [43]) were performed on the data for each trait. To test for associations between traits and ND5 deletion levels, we calculated Pearson correlation coefficients between trait values and isolate-specific ND5 deletion percentages, and applied one-way ANOVA to data categorized as being from high, low, or zero-ND5 deletion isolates (see Figure 1B legend). Finally, we characterized correlations among life-history characters measured on the same individuals by calculating Spearman rank correlation coefficients between each pair of traits following [26].

\section{Additional material}

Additional file 1: Table S1. ND5 deletion heteroplasmy data.

Additional file 2: Table S2. Life-history trait correlations.

\section{Acknowledgements}

We are deeply appreciative of the K. Garlid lab for willingness to share protocols and equipment, and of A. Snyder (Advanced Light Microscopy Core Facility, OHSU) and the B. Braekman lab for generous technical advice and assistance. We also thank H. Bui, S. Anderson, and T. Polley for laboratory assistance, and D. Howe and three anonymous reviewers for helpful suggestions on the manuscript. This work was supported by NSF grant DEB-0743871 (SE and DRD), PSU Faculty Enhancement Grant (SE), and PSU Undergraduate Scholarly and Creativity Grants (ACH and JK).

\section{Author details}

'Department of Biology, Portland State University, Portland, OR 97201, USA. 2Department of Zoology and Center for Genome Research and Biocomputing, Oregon State University, Corvallis, OR 97331, USA.

\section{Authors' contributions}

ALC-H and KAH carried out the metabolic and oxidative stress assays. KAH, SRM, JBK, and SWS carried out the life-history assays. KAH, KCS, and GdH carried out the reactive oxygen species assays. SE oversaw all experiments and data analysis, and drafted the manuscript, and DRD provided nematode stocks and helped draft the manuscript. All authors read and approved the final manuscript.

Received: 22 April 2010 Accepted: 12 January 2011

Published: 12 January 2011 
References

1. Dolgin ES, Félix MA, Cutter AD: Hakuna Nematoda: genetic and phenotypic diversity in African isolates of Caenorhabditis elegans and C. briggsae. Heredity 2008, 100:304-315.

2. Kelleher DF, de Carvalho CE, Doty AV, Layton M, Cheng AT, Mathies LD, Pilgrim D, Haag ES: Comparative genetics of sex determination: masculinizing mutations in Caenorhabditis briggsae. Genetics 2008, 178:1415-1429

3. Rockman MV, Kruglyak L: Recombinational landscape and population genomics of Caenorhabditis elegans. PLoS Genet 2009, 5:e1000419.

4. Anderson JL, Morran LT, Phillips PC: Outcrossing and the maintenance of males within C. elegans populations. J Hered 2010, 101:S62-S74.

5. Cutter AD, Weiang Y, Tsvetkov N, Sunil S, Félix MA: Molecular population genetics and phenotypic sensitivity to ethanol for a globally diverse sample of the nematode Caenorhabditis briggsae. Molec Ecol 2010, 19:798-809.

6. Baer CF, Shaw F, Steding F, Baumgartner M, Hawkins A, Houppert A, Mason N, Reed M, Simonelic K, Woodard W, Lynch M: Comparative evolutionary genetics of spontaneous mutations affecting fitness in rhabditid nematodes. Proc Natl Acad Sci 2005, 102:5785-5790.

7. Hillier LW, Miller RD, Baird SE, Chinwalla A, Fulton LA, Koboldt DC, Waterson RH: Comparison of C. elegans and C. briggsae genome sequences reveals extensive conservation of chromosome organization and synteny. PLOS Biol 2007, 5:e167.

8. Zhao Z, Boyle TJ, Bao Z, Murray Jl, Mericle B, Waterson RH: Comparative analysis of embryonic cell lineage between Caenorhabditis briggsae and Caenorhabditis elegans. Dev Biol 2008, 314:93-99.

9. Husson SJ, Landuyt B, Nys T, Baggerman G, Boonen K, Clynen E, Lindemans M, Janssen T, Schoofs L: Comparative peptidomics of Caenorhabditis elegans versus C. briggsae by LC-MALDI-TOF MS. Peptides 2009, 30:449-457.

10. Howe DK, Denver DR: Muller's ratchet and compensatory mutation in Caenorhabditis briggsae mitochondrial genome evolution. BMC Evol Biol 2008, 8:62

11. Raboin MJ, Timko A, Howe DK, Félix MA, Denver DR: Evolution of Caenorhabditis mitochondrial genome pseudogenes and Caenorhabditis briggsae natural isolates. Molec Biol Evol 2010, 27:1087-1096.

12. Wallace DC: A mitochondrial paradigm of metabolic and degenerative diseases, ageing, and cancer: a dawn for evolutionary medicine. Annu Rev Genet 2005, 39:397-407.

13. Biskup S, Moore DJ: Detrimental deletions: mitochondria, aging and Parkinson's disease. Bioessays 2006, 28:963-967.

14. Taylor RW, Turnbull DM: Mitochondrial DNA mutations in human disease. Nat Rev Gen 2005, 6:389-402.

15. Shigenaga MK, Hagen TM, Ames BN: Oxidative damage and mitochondrial decay in aging. Proc Natl Acad Sci USA 1994, 91:10771-10778

16. Kayser EB, Sedensky MM, Morgan PG: The effects of complex I function and oxidative damage on lifespan and anesthetic sensitivity in Caenorhabditis elegans. Mech Ageing Dev 2004, 125:455-464.

17. Sedensky MM, Morgan PG: Mitochondrial respiration and reactive oxygen species in mitochondrial aging mutants. Exp Gerontol 2006, 41:237-245.

18. Falk MJ, Zhang Z, Rosenjack JR, Nissim I, Daikhin E, Sedensky MM, Yudkoff M, Morgan PG: Metabolic pathway profiling of mitochondrial respiratory chain mutants in C. elegans. Mol Genet Metab 2008, 93:388-397.

19. Phillips N, Salomon M, Custer A, Ostrow D, Baer CF: Spontaneous mutational and standing genetic (co)variation at dinucleotide microsatellites in Caenorhabditis briggsae and Caenorhabditis elegans. Mol Biol Evol 2009, 26:659.

20. Howe DK, Baer CF, Denver DR: High rate of large deletions in Caenorhabditis briggsae mitochondrial genome mutation processes. Genome Biol Evol 2010, 1:29-38.

21. Rossignol R, Faustin B, Rocher C, Malgat M, Mazat JP, Letellier T: Mitochondrial threshold effects. Biochem J 2003, 370:751-762.

22. Walker DW, McColl G, Jenkins NL, Harris J, Lithgow GJ: Evolution of lifespan in C. elegans. Nature 2000, 405:296-297.

23. Estes S, Ajie BC, Lynch M, Phillips PC: Spontaneous mutational correlations for life-history, morphological and behavioral characters in Caenorhabditis elegans. Genetics 2005, 170:645-653.
24. Chen J, Senturk D, Wang JL, Müller HG, Carey JR, Caswell H, CaswellChen EP: A demographic analysis of the fitness cost of extended longevity in Caenorhabditis elegans. J Gerontol Series A 2007, 62:126-135.

25. Williams GC: Pleiotropy, natural selection, and the evolution of senescence. Evolution 1957, 11:398-411.

26. Huang C, Xiong C, Kornfeld K: Measurements of age-related changes of physiological processes that predict life span of Caenorhabditis elegans. Proc Natl Acad Sci USA 2004, 101:8084-8089.

27. Collins JJ, Huang C, Hughes S, Kornfeld K: The measurement and analysis of age-related changes in Caenorhabditis elegans. In The C. elegans Research Community, WormBook Edited by: WormBook 2008 [http://www. wormbook.org].

28. Rea SL, Johnson TE: A metabolic model for life span determination in Caenorhabditis elegans. Devel Cell 2003, 5:197-203.

29. Rea SL: Metabolism in the Caenorhabditis elegans Mit mutants. Exp Gerontol 2005, 40:841-849.

30. Braeckman BP, Houthoofd K, Vanfleteren JR: Intermediary metabolism. In The C. elegans Research Community, WormBook Edited by: WormBook 2009 [http://www.wormbook.org].

31. Dingley S, Polyak E, Lightfoot R, Ostrovsky J, Rao M, Greco T, Ischiropoulos H, Falk MJ: Mitochondrial respiratory chain dysfunction variably increases oxidant stress in Caenorhabditis elegans. Mitochondrion 2010, 10:125-36

32. Zielonka J, Kalyanaraman B: Hydroethidine- and MitoSOX-derived red fluorescence is not a reliable indicator of intracellular superoxide formation: another inconvenient truth. Free Radic Biol Med 2010, 48:983-1001.

33. Baer CF, Miyamoto MM, Denver DR: Mutation rate variation in multicellular eukaryotes: causes and consquences. Nature Rev Gen 2007, 8:619-631

34. Murphy MP: How mitochondria produce reactive oxygen species. Biochem J 2009, 417:1-13.

35. Cutter AD, Félix MA, Barrière Charlesworth D: Patterns of nucleotide polymorphism distinguish temperate and tropical wild isolates of Caenorhabditis briggsae. Genetics 2006, 173:2021-2031.

36. Dolgin ES, Charlesworth B, Baird SE, Cutter AD: Inbreeding and outbreeding depression in Caenorhabditis nematodes. Evolution 2007 61:1339-1352.

37. Gems D, Doonan R: Oxidative stress and ageing in the nematode Caenorhabditis elegans. In Ageing Medicine: Oxidative Stress in Ageing: From Model Systems to Human Diseases. Edited by: Miwa S, Keckman KB, Muller FL. Totowa, NJ: Humana Press; 2008:81-110.

38. Dowling DK, Simmons LW: Reactive oxygen species as universal constraints in life-history evolution. Proc $R$ SOC B 2009, 276:1737-1745.

39. Estes S, Phillips PC, Denver DR, Thomas WK, Lynch M: Mutation accumulation in populations of varying size: the distribution of mutational effects for fitness correlates in Caenorhabditis elegans. Genetics 2004, 166:1269-1279.

40. Chow DK, Glenn CF, Johnston JL, Goldberg IG, Wolkow CA: Sarcopenia in the Caenorhabditis elegans pharynx correlates with muscle contraction rate over lifespan. Exp Gerontol 2006, 41:252-260.

41. Braeckman BP, Houthoofd K, De Vreese A, Vanfleteren JR: Assaying metabolic activity in ageing Caenorhabditis elegans. Mech Ageing Dev 2002, 123:105-119.

42. Lee SS, Lee RY, Fraser AG, Kamath RS, Ahringer J, Ruvkun G: A systematic RNAi screen identifies a critical role for mitochondria in C. elegans longevity. Nat Genet 2003, 33:40-48.

43. Zar JH: Biostatistical Analysis. 4 edition. Upper Saddle River, NJ: Prentice Halli 1999

doi:10.1186/1471-2148-11-11

Cite this article as: Estes et al:: Natural variation in life history and aging phenotypes is associated with mitochondrial DNA deletion frequency in Caenorhabditis briggsae. BMC Evolutionary Biology 2011 11:11. 